\title{
Tetramethylpyrazine induces heme oxygenase- 1 expression and attenuates myocardial ischemia/reperfusion injury in rats
}

\author{
Shu-Ying Chen ${ }^{1}$, George Hsiao ${ }^{2}$, Hwong-Ru Hwang ${ }^{3}$, Pao-Yun Cheng ${ }^{4}$ \\ \& Yen-Mei Lee ${ }^{4, *}$ \\ ${ }^{1}$ Department of Nursing, Hung Kuang University, Taichung, Taiwan; ${ }^{2}$ Department of Pharmacology, Taipei \\ Medical University, Taipei, Taiwan; ${ }^{3}$ Division of Cardiology, Department of Medicine, Kaohsiung Veterans \\ General Hospital, Kaohsiung, Taiwan; ${ }^{4}$ Department of Pharmacology, National Defense Medical Center, \\ No.161, Section 6, Min-Chuan East Road, NeiHu 114, Taipei, Taiwan
}

Received 16 April 2006; accepted 13 June 2006

(C) 2006 National Science Council, Taipei

Key words: arrhythmias, HO-1, myocardial ischemia, neutrophils, oxidative stress, reperfusion injury, tetramethylpyrazine

\begin{abstract}
The accumulation of oxygen free radicals and activation of neutrophils are strongly implicated as pathophysiological mechanisms mediating myocardial ischemia/reperfusion injury. Heme oxygenase-1 (HO-1) has been reported to play a protective role in oxidative tissue injuries. In this study, the cardioprotective activity of tetramethylpyrazine (TMP), an active ingredient of Chinese medicinal herb Ligusticum wallichii Franchat, was evaluated in an open-chest anesthetized rat model of myocardial ischemia/reperfusion injury. Pretreatment with TMP (5 and $10 \mathrm{mg} / \mathrm{kg}$, i.v.) before left coronary artery occlusion significantly suppressed the occurrence of ventricular fibrillation. After $45 \mathrm{~min}$ of ischemia and $1 \mathrm{~h}$ of reperfusion, TMP ( 5 and $10 \mathrm{mg} / \mathrm{kg}$ ) caused a significant reduction in infarct size and induced HO-1 expression in ischemic myocardium. The HO inhibitor ZnPP $(50 \mu \mathrm{g} / \mathrm{rat})$ markedly reversed the anti-infarct action of TMP. Superoxide anion production in ischemic myocardium after 10 min reperfusion was inhibited by TMP. Furthermore, TMP (200 and $500 \mu \mathrm{M})$ significantly suppressed fMLP $(800 \mathrm{nM})$-activated human neutrophil migration and respiratory burst. In conclusion, TMP suppresses ischemia-induced ventricular arrhythmias and reduces the infarct size resulting from ischemia/reperfusion injury in vivo. This cardioprotective activity of TMP may be associated with its antioxidant activity via induction of HO-1 and with its capacity for neutrophil inhibition.

\section{Introduction}

The oxygen free-radical system has been implicated in the pathogenesis of myocardial ischemia/reperfusion $(\mathrm{I} / \mathrm{R})$ injury $[1,2]$. The oxygen radicals are generated by injured myocytes and endothelial cells in the ischemic zone, as well as neutrophils that enter the ischemic zone, and become activated on reperfusion. These oxygen

*To whom correspondence should be addressed. Phone: +886 2-87927877, Fax: + 886-2-87927877, E-mail: ymlee@mail. ndmctsgh.edu.tw

radicals exacerbate membrane damage, which leads to calcium loading. The neutrophils accumulate in the microcirculation, release inflammatory mediators, and contribute to microvascular obstruction and the no-reflow phenomenon in the reperfused myocardium [3]. In experimental settings, many free-radical scavengers or antioxidant, which interrupt peroxidation by a number of different mechanisms, have been suggested to use as a protection strategy against reperfusion injury [4]. Thus, a drug is capable of leukocyte inhibition and possesses antioxidant capacity may be useful in suppression of myocardial $\mathrm{I} / \mathrm{R}$ injury.
\end{abstract}


Heme oxygenase-1 (HO-1) has been reported to play a protective role in oxidative tissue injuries. The inhibition of $\mathrm{HO}$ activity and the suppression of ho-l gene expression lead to the aggravation of oxidative tissue injuries. In contrast, induction of HO-1 or exogenous administration of HO-1 by gene transfer conferred significant protection in a rat model of inflammation and in a hemoglobin-heme toxicity model [5]. Recently, HO-1 has been demonstrated to protect the heart. Yet et al. [6] reported that cardiac-specific transgenic mice overexpressing HO-1 provides potent cytoprotection against myocardial ischemia/reperfusion (I/R) injury. They also showed that HO-1 null mice exhibit increased right ventricular infacts and increased lipid peroxidation and oxidative damage in right ventricular cardiomyocytes when compared with wild-type $\mathrm{HO}$ mice [7]. Moreover, cardioselective overexpression of $\mathrm{HO}-1$ prevents $\mathrm{I} / \mathrm{R}$-induced cardiac dysfunction and apoptosis [8]. Induction of $\mathrm{HO}-1$ by hemin significantly reduces infarct size, oxidative stress and calcium overload induced by myocardial I/R [9]. Therefore, HO-1 expression in ischemic myocardium plays a crucial mediator of antioxidant and tissue protective actions.

Tetramethylpyrazine (TMP), an active ingredient of a traditional Chinese herb, Ligusticum wallichii Franch, has been widely used especially in the treatment of patients with cerebral and cardiac ischemic diseases in China [10]. Several pharmacological actions produced by TMP may contribute in attenuation of $I / R$ injury. TMP scavenges superoxide anions in human ploymorphonuclear leukocytes [11], attenuates ironinduced oxidative damage in cerebellar granule cell [12] and reduces lipid peroxidation in streptozotocin-induced diabetic mice [13]. In addition, TMP has been reported to block cell adhesion molecule P-selectin expression[14], as well as to diminish the up-regulation of ICAM-1 [15], which leads to attenuate neutrophil infiltration. Based on these results, in the present study, we investigated the cardioprotective effect of TMP in an open-chest anesthetized rat model of myocardial ischemia/reperfusion injury and its possible mechanisms.

\section{Methods}

\section{Animal preparation}

Sprague Dawley (SD) rats were obtained from the National Laboratory Animal Breeding and Research Center of the National Science Council, Taiwan. Handling of the animals was in accordance with the Guide for the Care and Use of Laboratory Animals published by the U.S. National Institutes of Health (NIH Publication No. 85-23, revised 1996). Adult SD rats (male; 280$330 \mathrm{~g}$ ) were anesthetized with intraperitoneal pentobarbital sodium $(50 \mathrm{mg} / \mathrm{kg})$. Tracheotomy was performed and an intubating cannula was connected to a rodent ventilator. The animals were ventilated artificially with room air. Respiratory rate was synchronized with the rat's spontaneous rate (50-60 strokes/min, $1 \mathrm{ml} / 100 \mathrm{~g}$ body weight). Arterial blood $\mathrm{pH}$ and blood gases were maintained within normal physiological limits (pH: 7.35-7.45; $\mathrm{P}_{\mathrm{CO}_{2}}: \quad 30-35 \mathrm{mmHg} ; \mathrm{P}_{\mathrm{O}_{2}}: 85-$ $100 \mathrm{mmHg}$ ) by adjusting the respiratory rate and tidal volume. The left femoral artery and vein were cannulated for measurements of arterial blood pressure and heart rate via a Statham pressure transducer and a Biotechnometer (RS3400, Gould, USA) and for administration of drugs, respectively. Electrocardiograms were recorded from standard lead II limb leads. An oscilloscope electrocardiogram monitor (DSO 420, Gould, USA) was used to display the electrocardiogram continuously throughout the experiment. All signals, including the electrocardiogram and hemodynamic data, were recorded.

After a left-side thoracotomy was performed at the fifth intercostal space, the pericardium was incised and the heart was exteriorized. A ligature (6/0 silk suture) was placed around the left main coronary artery close to its origin. The thread was then made into a knot as an occluder and another thread was tied to the former knot as a releaser. The ends of both threads were brought outside the thoracic cavity. Thus, the occlusion could be tightened or loosened by pulling the thread of the releaser. The rat was then allowed to stabilize for $30 \mathrm{~min}$. During this period, the rats that showed functional instability such as hypotension 
(systolic blood pressure value less than $100 \mathrm{mmHg}$ ) or occurrence of cardiac arrhythmias were discarded. The left coronary artery was occluded for $45 \mathrm{~min}$ followed by $1 \mathrm{~h}$ of reperfusion.

The animals were randomly assigned to one of five treatment groups at the beginning of the study. (1) control group: rats received the vehicle, dimethyl sulfoxide (DMSO i.v., $0.02-0.03 \mathrm{ml}$ ) 20 min before 45 occlusion ( $n=16$ ); (2) TMP-2 group: TMP $2 \mathrm{mg} / \mathrm{kg}$ was given as an intravenous bolus $20 \mathrm{~min}$ prior to occlusion $(n=7)$; (3) TMP-5 group: TMP $5 \mathrm{mg} / \mathrm{kg}$ was administered as an intravenous bolus $20 \mathrm{~min}$ prior to occlusion $(n=8)$; (4) TMP-10 group: TMP $10 \mathrm{mg} / \mathrm{kg}$ (i.v. bolus) was administered $20 \mathrm{~min}$ prior to occlusion $(n=10)$; (5) $\mathrm{ZnPP} / \mathrm{TMP}-10$ group: with $2 \mathrm{~h}$-pretreatment of a $\mathrm{HO}$ inhibitor zinc protoporphyrin (ZnPP; $50 \mu \mathrm{g}$ per rat, i.p.) [16], TMP $10 \mathrm{mg} / \mathrm{kg}$ (i.v. bolus) was administered $20 \mathrm{~min}$ prior to occlusion $(n=6)$. The blood pressure, heart rate and electrocardiograms were continuously monitored throughout the experimental period.

\section{Measurements}

\section{Hemodynamics \\ Measurements of heart rate and mean arterial blood pressure were performed in all groups at baseline, 5, 10 and $30 \mathrm{~min}$ after occlusion and 5, 30 and $60 \mathrm{~min}$ after reperfusion. An indirect index of myocardial oxygen consumption was provided by calculation of the product of the systolic blood pressure and heart rate.}

\section{Ventricular arrhythmias during ischemic period}

Ventricular arrhythmias, which occurred within $30 \mathrm{~min}$ of the onset of myocardial ischemia were assessed by the onset, incidence, total number of ventricular premature contractions (VPC), and total duration of ventricular tachycardia (VT) and ventricular fibrillation (VF). We defined VPC as discrete and identifiable premature QRS complexes (premature in relation to the $\mathrm{P}$ wave), VT as a run of six or more consecutive ventricular premature beats and $\mathrm{VF}$ as signals lacking identifiable individual QRS deflections and for which a rate could no longer be determined. The mortality in each group was also evaluated.
Area at risk and infarct

At the end of the experiments, the coronary artery was re-occluded and $0.5 \mathrm{ml}$ methylene blue $(3 \%)$ was injected intravenously to denote the area at risk. The heart was then excised and the atria were removed. The entire ventricular area was sectioned into four $2-3 \mathrm{~mm}$ thick slices from the apex to the base and incubated in $(0.1 \%)$ nitroblue tetrazolium chloride $\left(20 \mathrm{~min}, 37^{\circ} \mathrm{C}\right)$. This solution stained the normal myocardium purple while the infarct portion remained pale. The areas of risk and infarct were taken with digital camera. The areas were then measured by computerized planimetry.

\section{Superoxide anion production in myocardium}

Superoxide anion production in cardiomyocytes is measured by modified lucigenin-enhanced chemiluminescence. The chemical specificity of this lightyielding reaction for superoxide ion was reported previously $[17,18]$. Briefly, myocaridium samples (control and TMP-10 groups) $(2 \times 2 \mathrm{~mm})$ taken from the non-ischemic and ischemic regions after the insult of $45 \mathrm{~min}$ and $10 \mathrm{~min}$ reperfusion were placed in $37^{\circ} \mathrm{C}$ Krebs-HEPES buffer and allowed to equilibrate for $10 \mathrm{~min}$. Scintillation plates containing Krebs-HEPES buffer with lucigenin $(250 \mu \mathrm{M})$ were placed into a microplate luminometer (LB96V, EG\&G Berthold, Germany). After $20 \mathrm{~min}$, background counts were recorded and then a myocardium sample was added to each well. Counts were then recorded for $15 \mathrm{~min}$ for each well and the respective background was subtracted. All samples were dried in a $90{ }^{\circ} \mathrm{C}$ $(16 \mathrm{~h})$ oven for expressing results on a milligram myocardium dry weight basis. Lucigenin chemiluminescence is calibrated using known rates of superoxide production from $0.5 \mathrm{mU} / \mathrm{ml}$ xanthine oxidase plus $100 \mu \mathrm{M}$ of xanthine, as determined by cytochrome $\mathrm{C}$ reduction. The results are expressed as relative luminescence unit (RLU)/mg of dry tissue weight.

Western blot analysis of HO-1 protein expression To detect HO immunoreactive proteins, the ischemic region of myocardium was isolated and immediately frozen in liquid nitrogen, and stored at $-80{ }^{\circ} \mathrm{C}$ until processed. Myocardium was ground using a ultrasonic cell disruptor (MISONIX, MICROSON ${ }^{\mathrm{TM}}$, NY, USA) in eppendorff containing lysis buffer $(50 \mathrm{mM}$ Tris $-\mathrm{HCl}, 1 \mathrm{mM}$ EDTA, $1 \mathrm{mM}$ EGTA, and $1 \%$ Triton X-100, 
pH 7.4) with protease inhibitor cocktail (Sigma) and phosphatase inhibitor cocktail 1 (Sigma) and agitated for $1 \mathrm{~h}$ at $4^{\circ} \mathrm{C}$. After centrifugation at $10,000 \times g$ at $4^{\circ} \mathrm{C}$ for $30 \mathrm{~min}$, the pellet was discarded and the protein concentration in the supernatant determined using a dye-binding assay (Bio-Rad) with bovine serum albumin as the standard.

Samples were subjected to electrophoresis in a $10 \%$ sodium dodecyl sulfate-polyacrylamide gel for $2 \mathrm{~h}$ at $100 \mathrm{~V}$. The separated proteins were then transferred electrophoretically to a nitrocellulose membrane (Millipore) at $0.25 \mathrm{~A}$ for $2 \mathrm{~h}$ in transfer buffer $(25 \mathrm{mM}$ Tris[hydroxymethyl]aminomethane, $192 \mathrm{mM}$ glycine, 20\% methanol, $\mathrm{pH} 8.3$ ). The blot was blocked for $1.5 \mathrm{~h}$ at room temperature in Tris-buffered saline (TBS), pH 7.4, containing $0.1 \%$ Tween-20 and $5 \%$ skimmed milk. The blot was then incubated overnight at $4{ }^{\circ} \mathrm{C}$ with rabbit polyclonal antibody directed against HO-1 (1:1000 dilution; BioVision Inc., CA, USA) in TBS containing $0.1 \%$ Tween-20. The membrane was washed and incubated for $1 \mathrm{~h}$ at room temperature with horseradish-peroxidase-conjugated antimouse IgG antibody (1:1,700 dilution; Cell Signaling, MA, USA). After successive washes with TBST, the bound antibody was detected using a SuperSignal ${ }^{\circledR}$ West Femto chemiluminescent substrate (Pierce), and the membrane was exposed to $\mathrm{X}$-ray film for $10 \mathrm{~s}$. The density of the individual bands was quantified by densitometric scanning of the blots using Image-Pro software.

\section{Chemotaxis of neutrophil}

Neutrophils were isolated by sedimentation through dextran and Ficoll/Hypaque gradient centrifugation from healthy individuals as described [19]. The chemotaxis assay was performed by the 24-well transwell system (Corning, NY, USA). The chemoattractants of $N$-formylmethionyl-leucyl-phenylalanine (fMLP; $800 \mathrm{nM}$ ) or free buffer as negative control were placed in the wells of 24-well tissue culture plates. The transwell inserts ( $8 \mu \mathrm{m}$ pore size) were filled with neutrophils in modified HBSS and were placed into the solution of inserts. Both sides were added with indicated concentrations of TMP. The plate was incubated for $90 \mathrm{~min}$ in the culture incubator. The number of migrated neutrophils into the bottom of each wells were quantitated with inverted microscope (TS100, Nikon, Japan) equipped with phase- contrast objectives. Total migrated neutrophils were counted in four randomly selected microscope fields $(\times 10)$ and were also captured by digital image system (Image-Pro plus Software 4.5, MediaCybernectics, USA).

\section{Neutrophil oxidative burst activity}

Superoxide production of neutrophils was measured by the method of lucigenin-enhanced chemiluminescence as described by Hsiao et al. [19] with some modification. Washed neutrophil suspension $\left(2 \times 10^{6}\right.$ cells $\left./ \mathrm{ml}\right)$ in modified Hanks' balanced salt solution with addition of $1 \mathrm{mM} \mathrm{CaCl} 2$ and $0.5 \mathrm{mM} \mathrm{MgCl} 2$ was dispensed to the wells of a standard scintillation microplate (Berthold, Germany). Before the assay, the cells were preincubated with vehicle (DMSO) or different concentrations of test compound. Then, $20 \mu \mathrm{l}$ aliquots of lucigenin were added at a final concentration of $100 \mu \mathrm{M}$ by the jet-injection system. The basal LCL was recorded at $37^{\circ} \mathrm{C}$ for $1 \mathrm{~min}$ by a microplate luminometer (Orion, Berthold, Germany) and the cells were immediately stimulated with fMLP $(800 \mathrm{nM})$ also added by the automatic injector. The luminescent light was recorded continuously for $5 \mathrm{~min}$. The chemiluminescent signal was represented as relative light units per second (RLU/s). The results of lucigenin-enhanced chemiluminescence intensity (as increment in signal intensity) were determined by measuring basal and stimulator-induced peak values and calculating the difference between them.

\section{Statistical analysis}

The measurements of hemodynamics, total number of ventricular premature contractions, total duration of VT and VF, level of superoxide anion production, percentage of neutrophil migration, HO-1 protein expression, area at risk and infarct are expressed as group means \pm standard error (SE) of the mean. The Fisher's exact test was used to analyze the differences in the incidence of arrhythmias and mortality between the control and TMP-treated groups. The other parameters were compared by one-factor analysis of variance. If this analysis indicated significant differences among the group means, the control group was compared with each of the treatment groups by means of the Newman-Keuls method. A $p$ value of 
less than 0.05 was considered statistically significant.

\section{Results}

\section{Hemodynamic measurements}

The hemodynamic data including mean blood pressure, heart rate and rate-pressure product were observed. No significant changes in these measurements were found when the control was compared with each of four treatment groups throughout the experimental period.

\section{Myocardial ischemia-induced arrhythmias}

Occlusion of the left coronary artery invariably resulted in ventricular arrhythmias, commenced within 4-6 min of occlusion, manifesting as ventricular premature contractions (VPC), ventricular tachycardia (VT) and ventricular fibrillation (VF). Table 1 shows the effects of TMP on the onset and incidence of arrhythmias following coronary artery occlusion in the rat. All the rats in the control group developed arrhythmias during the 30-min post-ligation period. Pretreatment with TMP $2 \mathrm{mg} / \mathrm{kg}$ can not significantly prevent the occurrence of ventricular arrhythmias resulted from myocardial ischemia. However, TMP 5 and $10 \mathrm{mg} / \mathrm{kg}$ significantly suppressed the incidence of VF as compared to the control. Besides, TMP $10 \mathrm{mg} / \mathrm{kg}$ markedly delayed the onset of VF and reduced the mortality when compared with $62.5 \%$ in the control. In addition, TMP at 2, 5 and
$10 \mathrm{mg} / \mathrm{kg}$ reduced the numbers of VPC (Figure 1A). It caused a pronounced shortening of the total duration VT and VF at $10 \mathrm{mg} / \mathrm{kg}$ (Figure 1B, C). Pretreatment with the HO inhibitor ZnPP did not statistically reverse the antiarrhythmic action of TMP $10 \mathrm{mg} / \mathrm{kg}$ (Table 1). Also, even with $\mathrm{ZnPP}$, TMP $10 \mathrm{mg} / \mathrm{kg}$ was found to reduce the numbers of VPC and the duration of VF (Figure 1A, C).

\section{Infarct size}

All control and treated hearts showed clearly demarcated areas of infarction as a consequence of $45 \mathrm{~min}$ of ischemia followed by 1-h reperfusion, as assessed by the $p$-nitro-blue-tetrazolium staining technique. No significant differences in the area at risk, expressed as percentage of the total left ventricle, were noted among the groups (Figure 2). A significant reduction in infarct size, expressed as percentage of the area at risk was noted in two TMP-pretreatment groups ( 5 and $10 \mathrm{mg} / \mathrm{kg}$ ) when compared with the control (TMP $5 \mathrm{mg} / \mathrm{kg}$ : $44.8 \pm 2.7 \%$, TMP $10 \mathrm{mg} / \mathrm{kg}: 33.4 \pm 3.8 \%$ vs. control: $66.4 \pm 4.8 \%)(p<0.05)$. Two-hour pretreatment with $\mathrm{ZnPP}$ reversed the cardioprotective effect caused by TMP $10 \mathrm{mg} / \mathrm{kg}$ ( $\mathrm{ZnPP} / \mathrm{TMP}-10$ : $53.9 \pm 2.8 \%$ vs. control, $n=4 ; p>0.05)$.

\section{The effect of TMP on superoxide anion production in myocardium}

Myocardial superoxide anion production was measured in non-ischemic and ischemic regions of the control and TMP $(10 \mathrm{mg} / \mathrm{kg})$-pretreated groups

Table 1 The protective effect of tetramethylpyrazine (TMP) on ischemia-induced ventricular arrhythmias in anesthetized open-chest rats.

\begin{tabular}{|c|c|c|c|c|c|c|c|c|}
\hline \multirow[t]{2}{*}{ Treatment } & \multirow[t]{2}{*}{$N$} & \multicolumn{2}{|l|}{ VPC } & \multicolumn{2}{|l|}{ VT } & \multicolumn{2}{|l|}{$\mathrm{VF}$} & \multirow[t]{2}{*}{ Mortality $(\%)$} \\
\hline & & onset (min) & incidence $(\%)$ & onset (min) & incidence $(\%)$ & onset ( $\mathrm{min})$ & incidence $(\%)$ & \\
\hline Control & 16 & $5.3 \pm 0.2$ & 100 & $6.2 \pm 0.4$ & 94 & $7.3 \pm 0.4$ & 94 & 62.5 \\
\hline TMP-2 & 7 & $4.8 \pm 0.3$ & 100 & $5.1 \pm 0.3$ & 100 & $6.2 \pm 0.2$ & 71 & 57 \\
\hline TMP-5 & 8 & $5.0 \pm 0.5$ & 100 & $6.3 \pm 0.4$ & 88 & $7.3 \pm 0.6$ & $50^{*}$ & 50 \\
\hline TMP-10 & 10 & $6.0 \pm 0.5$ & 100 & $7.1 \pm 0.9$ & $40^{*}$ & $11.5 \pm 0.7^{*}$ & $30^{*}$ & $10^{*}$ \\
\hline $\mathrm{ZnPP} / \mathrm{TMP}-10$ & 6 & $5.5 \pm 0.2$ & 100 & $6.9 \pm 0.9$ & 83.3 & $7.5 \pm 0.3$ & 6.7 & 33.3 \\
\hline
\end{tabular}

$N$, number of rats; TMP-2, $-5,-10$ : pretreatment with TMP 2,5 , or $10 \mathrm{mg} / \mathrm{kg} 20 \mathrm{~min}$ prior to ischemia, respectively; ZnPP/TMP: a selective HO-1 inhibitor ZnPP was given $2 \mathrm{~h}$ before TMP $10 \mathrm{mg} / \mathrm{kg}$; VPC: ventricular premature contraction; VT: ventricular tachycardia; VF: ventricular fibrillation, ${ }^{*} p<0.05$ compared with the control group. 

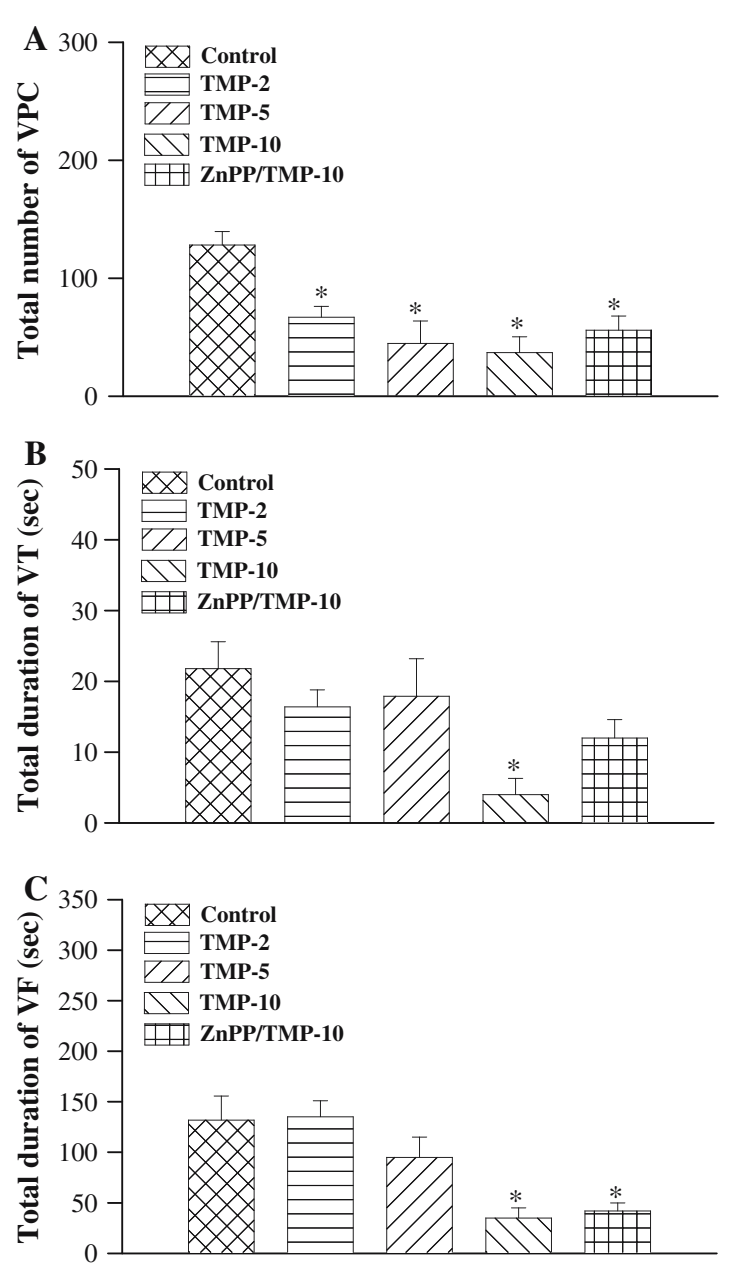

Figure 1. Effects of pretreatment with TMP on the total number of ventricular premature contractions (VPC) and the total duration of ventricular tachycardia (VT) and ventricular fibrillation (VF) during 45-min coronary artery occlusion in anesthetized rats. Values are expressed as mean $\pm \mathrm{SE}$, ${ }^{*} p<0.05$ vs. the control.

after 45-min ischemia/10-min reperfusion. Superoxide anion concentrations in the non-ischemic myocardium were similar in control and treatment groups $(p>0.05$, Figure 3$)$. Pretreatment with TMP $10 \mathrm{mg} / \mathrm{kg}$ prevented the increase in superoxide anion production in the myocardium after ischemia and reperfusion (TMP $10 \mathrm{mg} / \mathrm{kg}$ : $310 \pm 30$ vs. control: $687 \pm 20 \mathrm{RLU} / \mathrm{mg}$ tissue weight $)(p<0.05)$.

The effect of TMP on HO-1 protein expression in myocardium

HO-1 protein expression in the ischemic myocardium was studied by immunoblotting analysis.
Samples were collected after 45 min-ischemia and 1 h-reperfusion. Figure 4 shows that treatment with TMP $(10 \mathrm{mg} / \mathrm{kg})$ in non-ischemic myocardium for $2 \mathrm{~h}$ can induce marked HO-1 expression in myocardium. The level of HO-1 protein in the control group was quite low, whereas pretreatment with TMP 5 and $10 \mathrm{mg} / \mathrm{kg}$ significantly increased HO-1 protein expression in ischemic myocardium when compared with the control group $(p<0.05)$. HO-1 induction can last at least $2 \mathrm{~h}$ after reperfusion.

The effect of TMP on chemotaxis and respiratory burst of stimulated human neutrophils

TMP (100, 200 and $500 \mu \mathrm{M})$ significantly inhibited fMLP-induced chemotaxis of human neutrophils (Figure 5A), which was maximally inhibited at $200 \mu \mathrm{M}$ and did not show more inhibitory effect at $500 \mu \mathrm{M}$. However, no significant differences were found in the inhibitory effect of three concentrations of TMP.

fMLP stimulation of human neutrophils leads to the production of reactive oxygen species (ROS) by the neutrophil oxidase complex. The antineutrophil effect of TMP was evaluated by fMLP-lucigenin-dependent chemiluminescence. A rapid generation of superoxide anions was observed with lucigenin-dependent chemiluminescence signal up to $3040 \pm 207 \mathrm{RLU} / \mathrm{s}(n=13)$, when neutrophils were treated with fMLP $(800 \mathrm{nM})$. The increase of fMLP-induced chemiluminescnce was significantly inhibited by TMP 200 and $500 \mu \mathrm{M}(82.5 \pm 2.5$ and $57.0 \pm 3.2 \%$ of the control for 200 and $500 \mu \mathrm{M}$ TMP, respectively; $n=4)$.

\section{Discussion}

This is the first in vivo study demonstrating the cardioprotective effects of TMP on ischemic arrhythmias and reperfusion injury in rats. TMP markedly suppressed ventricular arrhythmias elicited by ischemia, and reduced infarct size and mortality. These effects may be achieved by its antioxidant activity via induction of HO-1 and by its capacity for neutrophil inhibition.

It is well known that oxygen free radicals are involved in the genesis of reperfusion injury. A substantial body of evidence has shown that 


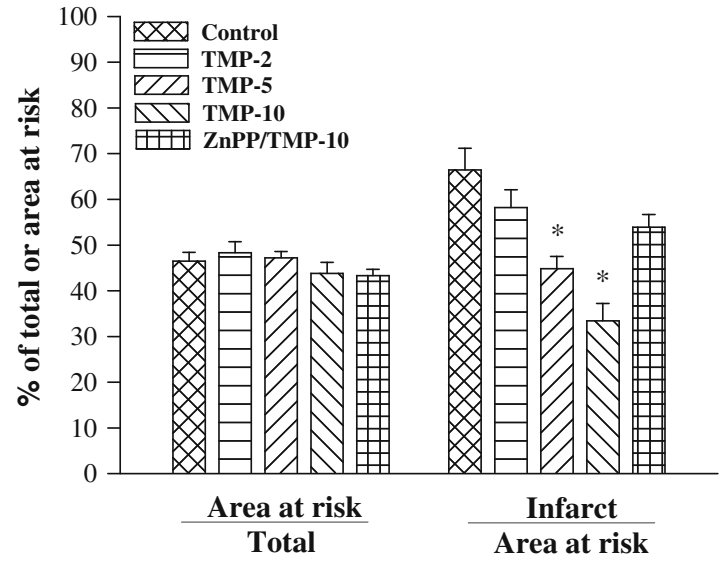

Figure 2. Effects of TMP on infarct size, expressed as a percentage of risk region, in rats undergoing $45 \mathrm{~min}$ of left coronary occlusion followed by $1 \mathrm{~h}$ of reperfusion. Values are expressed as mean $\pm \mathrm{SE},{ }^{*} p<0.05$ vs. the control.

interrupting peroxidation by antioxidants through a number of different mechanisms can prevent the production of oxygen free radicals [20]. In several reports show that TMP has a potent antioxidant activity [11-13]. In present study, we also showed that TMP suppressed superoxide anion production in myocardium exposed to I/R (Figure 3) and released by neurtrophils (Figure 5B). Thus, the cardioprotective effect of TMP may be related to prevent the production of free radicals.

Induction of HO-1 by TMP may contribute to the antioxidant activity. Without I/R, TMP

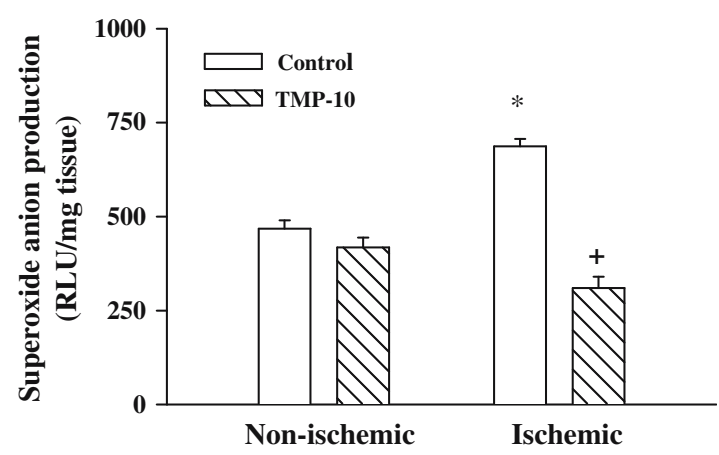

Figure 3. Effects of pretreatment with TMP $(10 \mathrm{mg} / \mathrm{kg})$ on superoxide anion production in myocardium exposed or not exposed to 45-min ischemia and 1-h reperfusion. RLU: relative luminescence unit; Values are expressed as mean \pm SE; $*: p<0.05$ vs. the non-ischemic region; $+: p<0.05$ vs. the control.
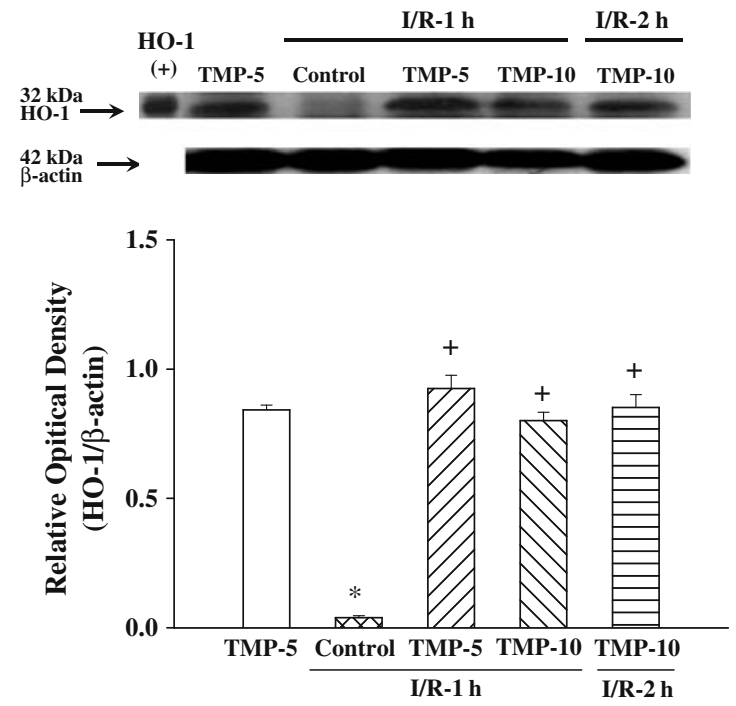

Figure 4. Effects of pretreatment with TMP on HO-1 expression in non-ischemic and ischemic myocardium exposed to 45-min ischemia and 1- or 2-h reperfusion. Representative Western blots are shown. HO-1 (+): HO-1 protein positive control; I/R-1 h: 45-min ischemia and 1-h reperfusion; I/R$2 \mathrm{~h}$ : 45-min ischemia and 2-h reperfusion; TMP-5, TMP-10: TMP 5 or $10 \mathrm{mg} / \mathrm{kg}$ was treated $20 \mathrm{~min}$ before myocardial ischemia. Values are expressed as mean $\pm \mathrm{SE} ; *: p<0.05$ vs. TMP alone, $+: p<0.05$ vs. the control.

treated in non-ischemic myocardium can induce cardiac HO-1 expression (Figure 4). In contrast, the HO-1 expression in the control group was not evident. This may be explained that the increased amount of free radicals after $I / R$ is not enough to induce $\mathrm{HO}-1$ in myocardium. TMP-induced HO-1 expression is beneficial to $\mathrm{I} / \mathrm{R}$ myocardium to reduce infarct size, which is proved by the reverse effect of $\mathrm{ZnPP}$, an $\mathrm{HO}$ inhibitor (Figure 2). Increased HO-1 expression leads to degradation of heme and accumulation of iron, bilirubin, and carbon monoxide $(\mathrm{CO})$ can reduce sensitivity of tissues to oxidant damage [21]. Of these metabolites, bilirubin acts as a direct antioxidant [22], whereas $\mathrm{CO}$ production helps to decrease vascular tone, inhibit platelet aggregation, and prevent both the production of pro-inflammatory cytokines and endothelial cell apoptosis [23]. Overexpression of HO-1 also has been demonstrated to provide potent cytoprotection against myocardial $\mathrm{I} / \mathrm{R}$ injury [6]. Therefore, HO-1 induction caused by TMP may contribute to reduce free radicals and lead to its anti-infarct effect. However, ZnPP did not completely prevent infarction caused by 

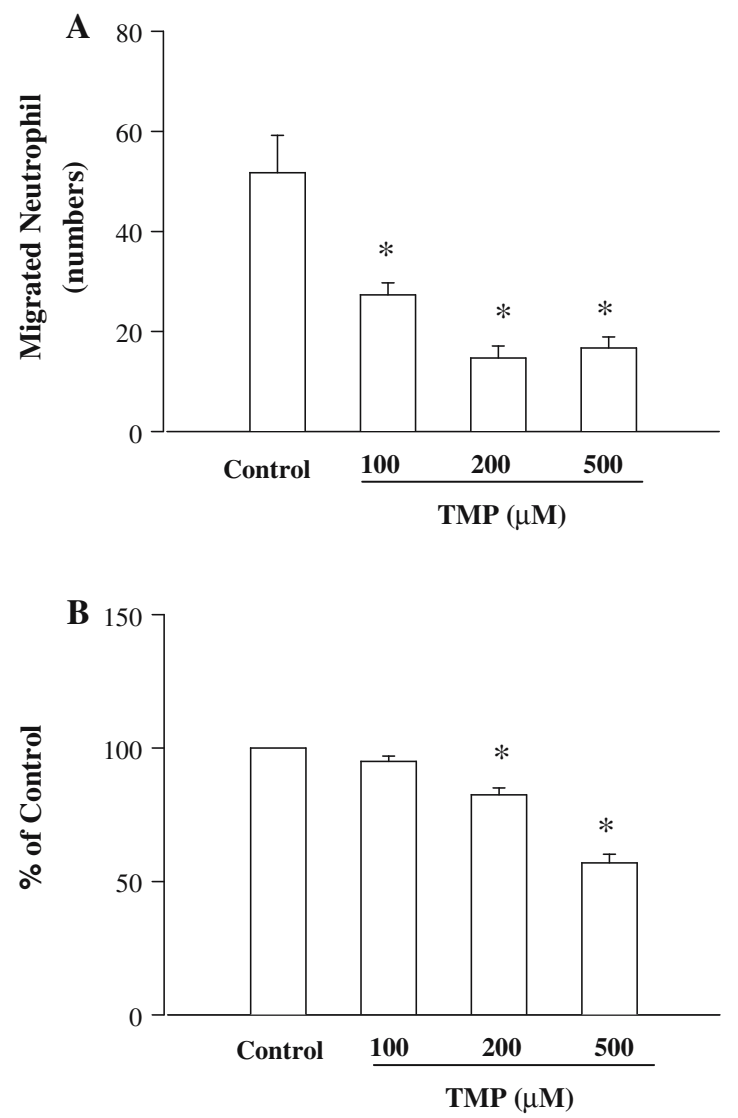

Figure 5. Effects of TMP on fMLP $(800 \mathrm{nM})$-stimulated chemotaxis (A) and respiratory burst (B) of human neutrophils. Values are expressed as mean \pm SE. ${ }^{*}: p<0.05$ vs. the control value.

I/R (Figure 2) indicating other mechanisms participating in the protective effect of TMP (see the followings).

Accumulating evidence has indicated that myocardial ischemia elicits an acute inflammatory response that is greatly augmented by reperfusion. Polymorphonuclear leukocytes are integrated into the acute inflammatory response to tissue injury. Polymorphonuclear leukocytes accumulate in ischemic and reperfused myocardium under the influence of chemoattractants, and participate in the myocardial injury after ischemia and reperfusion [24]. Activated polymorphonuclear leukocytes were shown to aggregate and adhere to endothelium, which resulted in capillary plugging and subsequent impairment of coronary blood flow and participation in the development of endothelial cell edema. Also, activated polymorphonuclear leukocytes exacerbate ischemic myocardial injury by release of cytotoxic oxygen free-radicals and proteolytic enzymes. TMP has been reported to block P-selectin expression [14] and to diminish the up-regulation of ICAM-1 [15] induced by I/R. Therefore, in the present study, the inhibitory effect of TMP on migration and respiratory burst of activated neutrophils may contribute to protect myocardium against $\mathrm{I} / \mathrm{R}$.

The pronounced cardioprotection by TMP is not likely to be mediated through its hemodynamic effects because no significant changes in mean blood pressure, heart rate or rate-pressure product occurred during experimental periods when compared with the control group. TMP did not alter the rate-pressure product, an index of myocardial oxygen consumption, indicating that reduced oxygen consumption cannot account for the antiarrhythmic effect. However, the therapeutic effect of TMP may be related to the following evidence: (i) TMP can cause coronary vasodilation in the dog [25, 26] and pig [27]. (ii) TMP possesses antiplatelet activity in rat thrombotic model $[28,29]$, which can result in improvement of microcirculation by attenuation of microembolism. Platelet products, e.g. TXA and serotonin, may exacerbate microcirculatory spasm, leading to further thrombosis and sluggish coronary flow [4]. (iii) TMP inhibits the L-type $\mathrm{Ca}^{2+}$ currents in rat ventricular myocytes [30]. In cultured vascular smooth muscle cells, TMP can open ATP-sensitive potassium channels and/or small conductance calcium-activated potassium channels to decrease intracellular $\mathrm{Ca}^{2+}$ concentration $\left[\mathrm{Ca}^{2+}\right]_{i}[31]$. Changes in intracellular $\mathrm{Ca}^{2+}$ homeostasis play an important role in the development of myocardial $\mathrm{I} / \mathrm{R}$ injury. Ischemia and reperfusion are both associated with an increase in $\left[\mathrm{Ca}^{2+}\right]_{i}$, which will contribute to the ischemia-induced ventricular arrhythmias and lead to reperfusion injury [4]. (iv) TMP has been reported to increases $\mathrm{PGI}_{2}$ and diminishes $\mathrm{TXA}_{2}$ release in the hypoxic isolated rat heart [32]. $\mathrm{TXA}_{2}$ is released from the myocardium in ischemia which contributes to the severity of the arrhythmias, whereas $\mathrm{PGI}_{2}$ is also generated during myocardial ischemia and possesses antiarrhythmic actions [33]. However, pretreatment with ZnPP did not reverse the anti-arrhythmic action of TMP (Table 1, Figure 1A, C) indicating 
that the anti-arrhythmic effect of TMP is not mediated through the action of HO-1. Furthermore, TMP shows the anti-apoptotic effect on cerebellar granule cells by suppression of oxidative stress [12]. According to the results by Fliss and Gattinger, apoptosis can be found in the myocardium of rats after $45 \mathrm{~min}$ ischemia and $2 \mathrm{~h}$ of reperfusion [34]. As the induction of HO-1 lasted for $2 \mathrm{~h}$ reperfusion, TMP may prevent apoptosis elicited by $I / R$ via attenuation of oxidative stress.

In conclusion, TMP has pronounced cardioprotective activity in a rat model of myocardial I/ $\mathrm{R}$. We postulated that the cardioprotective effects of TMP could be attributed to its capacity for neutrophil inhibition and to its antioxidant property via induction of HO-1.

\section{Acknowledgements}

This study was supported by research grant from the Nation Science Council (NSC93-2320-B016-039 to YM Lee), Taipei, Taiwan, ROC.

\section{References}

1. Maxwell S.R.J. and Lip G.Y.H., Reperfusion injury: a review of the pathophysiology, clinical manifestations and therapeutic options. Int. J. Cardiol. 58: 95-117, 1997.

2. Park J.L. and Lucchesi B.R., Mechanisms of myocardial reperfusion injury. Ann. Thorac. Surg. 68: 1905-1912, 1998.

3. Buja L.M., Myocardial ischemia and reperfusion injury. Cardiovasc. Pathol. 14: 170-175, 2005.

4. Moens A.L., Claeys M.J., Timmermans J.P. and Vrints C.J., Myocardial ischemia/reperfusion-injury, a clinical view on a complex pathophysiological process. Int. J. Cardiol. 100: 179-190, 2005.

5. Takahashi T., Morita K., Akagi R. and Sassa S., Heme Oxygenase-1: a novel therapeutic target in oxidative tissue injuries. Curr. Med. Chem. 11: 1545-1561, 2004.

6. Yet S.F., Tian R., Layne M.D., Wang Z.Y., Maemura K., Solovyeva M., Ith B., Melo L.G., Zhang L., Ingwall J.S., Dzau V.J., Lee M.E. and Perrella M.A., Cardiac-specific expression of heme oxygenase-1 protects against ischemia and reperfusion injury in transgenic mice. Circ. Res. 89: 168-173, 2001.

7. Yet S.F., Perrella M.A., Layne M.D., Hsieh C.M., Maemura K., Kobzik L., Wiesel P., Christou H., Kourembanas S. and Lee M.E., Hypoxia induces severe right ventricular dilatation and infarction in heme oxygenase-1 null mice. J. Clin. Invest. 103: R23-R29, 1999.

8. Vulapalli S.R., Chen Z., Chua B.H., Wang T. and Liang C.S., Cardioselective overexpression of HO-1 prevents I/Rinduced cardiac dysfunction and apoptosis. Am. J. Physiol. 283: H688-H694, 2002.
9. Masini E., Vannacci A., Marzocca C., Pierpaoli S., Giannini L., Fantappie O., Mazzanti R. and Mannaioni P.F., Heme oxygenase-1 and the ischemia-reperfusion injury in the rat heart. Exp. Biol. Med. 228: 546-549, 2003.

10. Guo S.K., Chen K.J., Qian Z.H., Weng W.L. and Qian M.Y., Tetramethylpyrazine in the treatment of cardiovascular and cerebrovascular diseases. Planta Medica 47: 89, 1983.

11. Zhang Z., Wei T., Hou J., Li G., Yu S. and Xin W., Tetramethylpyrazine scavenges superoxide anion and decreases nitric oxide production in human polymorphonuclear leukocytes. Life Sci. 72: 2465-2472, 2003.

12. Zhang Z., Wei T., Hou J., Li G., Yu S. and Xin W., Ironinduced oxidative damage and apoptosis in cerebellar granule cells: attenuation by tetramethylpyrazine and ferulic acid. Eur. J. Pharmacol. 467: 41-47, 2003.

13. Lee L.M., Liu C.F. and Yang P.P., Effect of tetramethylpyrazine on lipid peroxidation in streptozotocin-induced diabetic mice. Am. J. Chin. Med. 30: 601-608, 2002.

14. Chen J.L., Zhou T., Chen W.X., Zhu J.S., Chen N.W., Zhang M.J. and Wu Y.L., Effect of tetramethylpyrazine on P-selectin and hepatic/renal ischemia and reperfusion injury in rats. World J. Gastroenterol. 9: 1563-1566, 2003.

15. Feng L., Xiong Y., Cheng F., Zhang L., Li S. and Li Y., Effect of ligustrazine on ischemia-reperfusion injury in murine kidney. Transplant. Proc. 36: 1949-1951, 2004.

16. Fujimoto H., Ohno M., Ayabe S., Kobayashi H., Ishizaka N., Kimura H., Yoshida K. and Nagai R., Carbon monoxide protects against cardiac ischemia-reperfusion injury in vivo via MAPK and Akt-eNOS pathways. Arterioscler. Thromb. Vasc. Biol. 24: 1848-1853, 2004.

17. Gyllenhammer H., Lucigenin chemiluminescence in the assessment of neutrophil superoxide production. J. Immunol. Methods 97: 209-213, 1987.

18. Lee Y.M., Hsiao G., Chen H.R., Chen Y.C., Sheu J.R. and Yen M.H., Magnolol reduces myocardial ischemiarreperfusion injury via neutrophil inhibition in rats. Eur. J. Pharmacol. 422: 159-167, 2001.

19. Hsiao G., Teng C.M., Sheu J.R., Cheng Y.W., Lam K.K., Lee Y.M., Wu T.S. and Yen M.H., Cinnamophillin as a novel antiperoxidative cytoprotectant and free radical scavenger. Biochim. Biophys. Acta 1525: 77-88, 2001.

20. Kukreja R.C. and Hess M.L., The oxygen free radical system: from equations through membrane-protein interactions to cardiovascular injury and protection. Cardiovasc. Res. 26: 641-655, 1992.

21. Maines M.D., The heme oxygenase system: a regulator of second messenger gases. Annu. Rev. Pharmacol. Toxicol. 37: 517-554, 1997.

22. Stocker R., Yamamoto Y., McDonagh A.F., Glazer A.N. and Ames B.N., Bilirubin is an antioxidant of possible physiological importance. Science 235: 1043-1046, 1987.

23. Wagener F.A., Volk H.D., Willis D., Abraham N.G., Soares M.P., Adema G.J. and Figdor C.G., Different faces of the heme-heme oxygenase system in inflammation. Pharmacol. Rev. 55: 551-571, 2003.

24. Jordan J.E., Zhao Z.Q. and Vinten-Johansen J., The role of neutrophils in myocardial ischemia-reperfusion injury. Cardiovasc. Res. 43: 860-878, 1999.

25. Dai X.Z. and Bache R.J., Coronary and systemic haemodynamic effect of tetramethylpyrazine in the dog. J. Cardiovasc. Pharmacol. 7: 841-849, 1985.

26. Lin C.I., Wu S.L., Tao P.L., Chen H.M. and Wei J., The role of cyclic AMP and phosphodiesterase activity in the 
mechanism of action of tetramethylpyrazine on human and dog cardiac and dog coronary arterial tissues. J. Pharm. Pharmacol. 45: 963-966, 1993.

27. Shan Au A.L., Kwan Y.W., Kwok C.C., Zhang R.Z. and He G.W., Mechanisms responsible for the in vitro relaxation of ligustrazine on porcine left anterior descending coronary artery. Eur. J. Pharmacol. 468: 199-207, 2003.

28. Liu S.Y. and Sylvester D.M., Antithrombotic/antiplatelet activity of tetramethylpyrazine. Thromb. Res. 58: 129-140, 1990.

29. Liu S.Y. and Sylvester D.M., Antiplatelet activity of tetramethylpyrazine. Thromb. Res. 75: 51-62, 1994.

30. Zou L.Y., Hao X.M., Zhang G.Q., Zhang M., Guo J.H. and Liu T.F., Effect of tetramethyl pyrazine on L-type calcium channel in rat ventricular myocytes. Can. J. Physiol. Pharmacol. 79: 621-626, 2001.

31. Wong K.L., Chan P., Huang W.C., Yang T.L., Liu I.M., Lai T.Y., Tsai C.C. and Cheng J.T., Effect of tetramethylpyrazine on potassium channels to lower calcium concentration in cultured aortic smooth muscle cells. Clin. Exp. Pharmacol. Physiol. 30: 793-798, 2003.

32. Feng J., Liu R., Wu G. and Tang S., Effects of tetramethylpyrazine on the release of $\mathrm{PGI}_{2}$ and $\mathrm{TXA}_{2}$ in the hypoxic isolated rat heart. Mol. Cell. Biochem. 167: 153-158, 1997.

33. Parratt J., Endogenous myocardial protective (antiarrhythmic) substances. Cardiovasc. Res. 27: 693-702, 1993.

34. Fliss H. and Gattinger D., Apoptosis in ischemic and reperfused rat myocardium. Circ. Res. 79: 949-956, 1996. 